\title{
Análisis de variables de sitio para estimar el establecimiento en Chile de Acacia melanoxylon R. Br.
}

\author{
Analysis of site variables to estimate the establishment in Chile of Acacia melanoxylon $\mathrm{R}$. Br.
}

\author{
JUAN CARLOS RAMIREZ, JUAN SCHLATTER
}

Instituto de Silvicultura, Universidad Austral de Chile,

Casilla 567, Valdivia, Chile.

\begin{abstract}
SUMMARY
Acacia melanoxylon R. Br. possesses interesting wood characteristics which make it an important species to establish in Chile, due to the high prices it reaches in the international markets. The present study defines the possible distribution of the species in Chile. For wood production, its geographical extension ranges between $37^{\circ} \mathrm{S}$ and $42^{\circ} 30^{\prime} \mathrm{S}$, from the Pacific Coast to the Andean mountain range. The most favourable region to establish $A$. melanoxylon plantations is the coastal zone of the VIII Region, as well as coastal and interior zones of the IX and $\mathrm{X}$ regions, below $400 \mathrm{~m}$ s.n.m. and with an absence of extreme minimal temperatures. The main limiting factor northwards of its potential distribution is the length of the dry period, while southwards the minimal temperature and frosts are the most limiting.
\end{abstract}

Key words: Acacia melanoxylon, potential distribution in Chile, site variables.

\section{RESUMEN}

La especie Acacia melanoxylon R. Br. posee interesantes características para su establecimiento en Chile, debido al alto precio que alcanza su madera en el mercado internacional. El presente trabajo delimitó la distribución potencial de la especie en Chile, siendo recomendable su establecimiento, con fines de producción maderera, desde sectores costeros y precordilleranos de la VIII Región ( $\left.37^{\circ} \mathrm{S}\right)$ hasta el límite sur de la X $\left(42^{\circ} 30^{\prime}\right)$. Se estimó como muy favorable para el establecimiento de plantaciones la zona costera de la VIII Región, así como zonas costeras e interiores de las IX y X Región, a elevaciones menores de 400 m s.n.m. y sin temperaturas mínimas extremas. Se concluyó que el principal factor limitante hacia el norte de la distribución potencial es la duración del período seco, mientras que hacia el sur lo son las temperaturas mínimas y la presencia de heladas.

Palabras claves: Acacia melanoxylon, distribución potencial en Chile, variables de sitio.

\section{INTRODUCCION}

El aromo australiano, Acacia melanoxylon $\mathrm{R}$. Br., es una especie leguminosa de la familia Mimosaceae, originaria de Australia. Se le considera una de las especies del género Acacia de mayor dimensión en el mundo, alcanzando más de $30 \mathrm{~m}$ de altura y diámetros de $>1 \mathrm{~m}$ en su habitat natural (De Zwaan 1982; Boland et al. 1992).

La especie posee un rápido crecimiento inicial y gran producción de semillas, con germinación cercana a $100 \%$, lo que le otorga gran capacidad colonizadora (Forest Research Institute 1982;
Kannegiesser 1989). Por ser además una especie leguminosa, es capaz de fijar el nitrógeno atmosférico, con lo cual contribuye a la recuperación de suelos.

La distribución natural del aromo australiano se asocia principalmente con sectores costeros del SE de Australia y el NO de la isla de Tasmania. Se ubica aproximadamente entre las latitudes $16^{\circ} \mathrm{y}$ $43^{\circ} \mathrm{S}$, hasta una distancia de $200 \mathrm{~km}$ de la costa (Wilkins y Papassotiriou 1989). La zona $\mathrm{N}$ de su distribución natural posee un clima subtropical a tropical, donde las abundantes precipitaciones alcanzan su máximo en verano. En la parte central 
el clima es templado cálido, mientras que en el $\mathrm{S}$ es templado frío. A lo largo de su distribución, la precipitación media anual varía entre 450 a 1800 $\mathrm{mm}$. En el centro las precipitaciones tienen una distribución uniforme y en el sur éstas presentan un máximo invernal (Farrell y Ashton 1978). Las temperaturas medias máximas oscilan entre $23^{\circ} \mathrm{C}$ y $26^{\circ} \mathrm{C}$ y las mínimas entre $1^{\circ} \mathrm{C}$ y $10^{\circ} \mathrm{C}$ (Boland et al. 1992, CONAF 1991).

Wilkins y Papassotiriou (1989) afirman que con el aumento de la latitud existe una tendencia hacia condiciones ambientales xerofíticas (inviernos fríos y veranos secos), especialmente en Tasmania. Kannegiesser (1989) menciona que el mejor desarrollo y calidad de la madera se alcanza en el $\mathrm{S}$ de Australia y NO de Tasmania, en este último caso en tierras bajas, con alta humedad la mayor parte del año.

La especie es exigente en agua (Esterhuyse 1985), afectándola períodos secos muy prolongados si no tiene humedad adicional en el suelo. Una buena calidad de madera se obtiene principalmente en condiciones de sitio favorables (Harrison $1975)$.

La madera de la especie puede alcanzar un alto precio en el mercado internacional, lo que invita a su establecimiento en plantaciones. La estética de la madera es muy atractiva para fines tales como ebanistería y revestimientos decorativos. En el año 1990 la madera aserrada se cotizó en US\$ 130 por $\mathrm{m}^{3}$ y en US\$ 550 por $\mathrm{m}^{3}$ la madera libre de nudos (CONAF 1991). Se alcanzan también precios ocasionales superiores a U\$ 1000 por $\mathrm{m}^{3}$ en trozas de gran diámetro y de calidad excepcional en cuanto a duraminización, sanidad y color. Según De Zwaan (1982), el factor más importante en la determinación del precio de la madera es el color del duramen, prefiriéndose maderas oscuras, ojalá de tonalidad homogénea. También se prefieren trozos cilindricos y sin defectos tales como nudos o arqueaduras, lo que significa la necesidad de un control silvicultural adecuado.

En Chile el aromo australiano ha sido plantado desde los $33^{\circ}-42^{\circ} \mathrm{S}$ con un éxito muy variado. En el norte predomina como árbol decorativo, en jardines y parques, y en el sur ha sido introducido como especie forestal complementaria a la agricultura. El desarrollo como árbol de dimensiones considerables lo ha logrado especialmente en el sur.

La especie ha despertado el interés en el sector forestal, como una posibilidad dentro de los pro- gramas de diversificación de especies forestales maderables (Lagos 1994). Su importancia y la información dispersa sobre ella motivaron el desarrollo del presente trabajo, con los siguientes objetivos:

- Identificar en base a la información bibliográfica disponible los principales requerimientos ambientales de la especie y la forma en que las variables de sitio pueden influir en su desarro110 .

- Inferir la distribución potencial de $A$. melanoxylon desde las características ambientales de Chile.

- Determinar las zonas del país donde la especie pueda encontrar condiciones más favorables para su desarrollo, especialmente como especie maderable.

\section{MATERIAL Y METODOS}

EVALUACION DE LA INFORMACION BIBLIOGRAFICA. A través de una recopilación bibliográfica accesible en Chile, se delimitó la distribución natural de aromo australiano en Australia (Farrell y Ashton, 1978), ocupando un mapa climático de Walter y Lieth (1960).

CARACTERIZACION CLIMATICA DEL AREA GEOGRAFICA CON LA ESPECIE. En el área geográfica con presencia de la especie se diferenciaron una región continental y otra insular (Tasmania, más oceánica), las cuales se subdividieron a su vez en distintas zonas y enclaves climáticos. En el mapa de la figura 1 la región continental se dividió en la zona A y los enclaves C, D, B, E y F, y la región insular se definió como zona $\mathrm{G}$.

Datos de estaciones meteorológicas en cada zona, enclave, distrito y áreas climáticas que acompañaban al mapa arriba indicado, permitieron obtener la información climática para caracterizar al área geográfica con aromo. La región continental, en su zona A, se subdividió para este efecto en los distritos Norte, Centro-Norte, Centro y Oeste. Complementariamente se distinguieron las características climáticas para los límites geográficos que indican condiciones umbrales para el aromo.

IDENTIFICACION DE LAS VARIABLES DE SITIO MAS IMPORTANTES PARA EL DESARROLLO DE AROMO AUSTRALIANO. Una revisión bibliográfica permitió seleccionar aquellas variables de sitio que son 


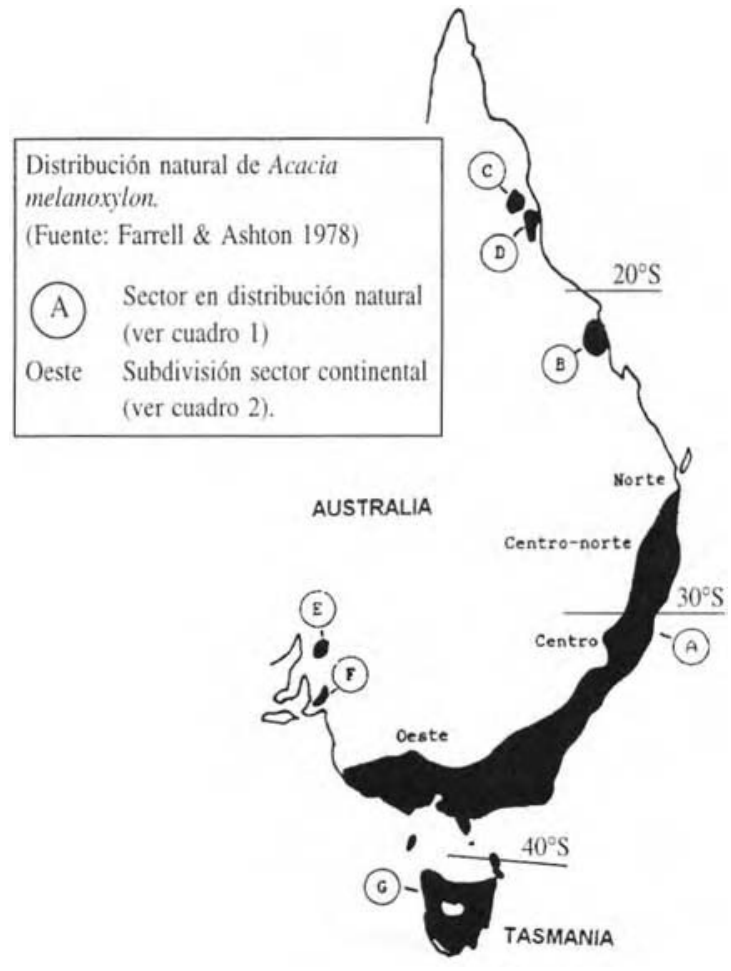

Figura 1. Distribución natural de Acacia melanoxylon. Natural distribution of Acacia melanoxylon.

las más influyentes en el desarrollo de aromo australiano. A cada variable climática seleccionada se adscribieron atributos cuantitativos, de acuerdo a su proximidad a los valores extremos para la especie (valores óptimos y valores límites), y de acuerdo a la especificación de otros autores. Estos rangos fueron calificados como: favorable, adecuado, moderado y desfavorable (cuadro 3 ).

Para las variables del suelo se establecieron criterios cualitativos, de acuerdo a su efecto sobre la especie. En este caso sólo se definieron dos niveles o rangos: favorable y desfavorable, debido a la falta de datos cuantitativos en la bibliografía.

\section{DISTRIBUCION POTENCIAL EN CHILE DE AROMO} AUSTRALIANO. Un análisis de los requerimientos de sitio previamente deducidos para la especie permitió comparar a éstos con la información contenida en el Atlas Agroclimático del INIA (1989). Esta información se complementó con el Atlas Agroclimático de Santibáñez y Uribe (1993) y el Sistema de Ordenamiento Tierra de Schlatter et al. (1994, 1995). El análisis se centró en las regio- nes del país donde se estima que se encuentran los límites potenciales para la especie en Chile, según CONAF (1995).

De acuerdo al tipo y número de variables ambientales que cumplen o no con los requerimientos de Acacia melanoxylon, se definieron cuatro categorías de calidad de sitio. Estas son:

- Categoría $N^{\circ}$ 1: Todas las variables climáticas se encuentran dentro del rango adecuado para la especie.

- Categoría $\mathrm{N}^{\circ}$ 2: Existe una de las variables del suelo fuera de los rangos de tolerancia, ésta puede sin embargo ser compensada por las demás condiciones ambientales.

- Categoría $\mathrm{N}^{\circ}$ 3: Existe una de las variables climáticas fuera de los rangos de tolerancia, la que puede ser compensada por las demás variables ambientales. El establecimiento de la especie, sin embargo, debe ser más selectivo en cuanto al sitio.

- Categoría $N^{\circ}$ 4: Existe más de una variable de sitio fuera de los rangos aceptados por la especie. Aquí sólo existen posibilidades de un establecimiento puntual. Esta categoría marca el límite de la distribución potencial de la especie. Estas categorías fueron aplicadas geográficamente utilizando el Sistema de Ordenamiento de la Tierra de Schlatter et al. (1994, 1995), el cual divide el territorio comprendido entre las regiones VII y $\mathrm{X}$ en unidades territoriales afines según sus características climáticas y de suelo. Las divisiones son las siguientes:

- Zona de crecimiento: Sector determinado por la variación del clima en sentido longitudinal. Se definen las zonas de crecimiento Cordillera de la Costa, Secano Interior, Depresión Intermedia, Precordillera Andina, Cordillera Andina y Zona Trasandina.

- Distrito de crecimiento: División de una zona de crecimiento, fundamentada en la variación climática en sentido de la latitud.

- Área de crecimiento: Sector de un distrito, determinado tanto por la variación climática en sentido altitudinal, así como también por el material de origen del suelo.

Para las áreas geográficas no consideradas por el trabajo de Schlatter et al. (1994, 1995) se utilizó la información correspondiente a los distritos climáticos determinados por INIA (1989) y Santibáñez y Uribe (1993). 
VALORACION DE LA CALIDAD DE SITIO PARA AROMO AUSTRALIANO. Con el fin de especificar la extensión geográfica potencial de la especie en Chile, se desarrolló un sistema cuantitativo para distinguir los sectores en donde la especie puede alcanzar un crecimiento adecuado, considerando los rangos de tolerancia del aromo a cada variable ambiental identificada previamente.

El sistema cuantitativo descansa en la adscripción de puntos a las variables climáticas de acuerdo a la calidad de su efecto sobre el aromo:

- Variable en rango favorable:

3 puntos;

- Variable en rango adecuado:

2 puntos;

- Variable en rango moderado:

1 punto;

- Variable en rango desfavorable: 0 punto.

La valorización de las variables de suelo utiliza el mismo sistema de puntos, pero con valores cualitativos según Schlatter et al. (1994 y 1995).

Sin embargo, cada factor o variable de sitio tiene un peso distinto en su efecto sobre las especies vivas (Schlatter y Gerding 1995), lo que necesariamente debe ser considerado en valoraciones de sitios. Por tal motivo se seleccionaron factores de corrección para considerar este aspecto, de acuerdo al efecto de las distintas variables. Se mantuvo la ponderación de las variables del suelo, por su menor peso regional. Pero entre las variables climáticas, aquellas relacionadas al régimen de temperatura obtuvieron una ponderación de 2 (se duplica) y las variables del régimen hídrico obtuvieron una ponderación de 3 (se triplica), por ser estas variables las más decisivas en la existencia y el desarrollo de la especie en un lugar geográfico determinado, especialmente en este caso en las regiones naturales en Australia y en la zona de interés en Chile.

La valoración de la calidad de sitio se realizó sumando los puntos asignados a las variables de sitio, en cada una de las áreas de crecimiento del Sistema de Ordenamiento de la Tierra (Schlatter et al. 1994 y 1995). Como se desprende del criterio de diagnóstico anterior, una mayor cantidad de puntos indicará un sitio potencialmente más favorable para el desarrollo de la especie. Sin embargo, en este procedimiento existe la posibilidad de que resulten una mayor cantidad de puntos en áreas con presencia de factores limitantes para la especie, en comparación a una sin limitantes, debido a la compensación que resulta por la acción de otras variables en condición favorable.

Para facilitar el análisis de los resultados del presente trabajo, se entrega a continuación la es- cala de calidades de sitio definida, considerando la cantidad de puntos totales:

- sitio excelente: sobre 39 puntos;

- sitio bueno: entre 35 y 39 puntos;

- sitio de mediana calidad: entre 30 y 34 puntos;

- sitio limitado: bajo 30 puntos.

Una de las variables más importantes del régimen hídrico es la longuitud del período seco (período en que la precipitación no alcanza a cubrir el $50 \%$ de la evapotranspiración potencial). Sin embargo, debido a la alta humedad relativa existente en los sectores costeros de Chile, el efecto del periodo seco es compensado en parte por ella. En el análisis de estimación de calidad de sitio se reemplazó por tal motivo el período seco por el índice de humedad anual (cuociente entre la precipitación anual y la evapotranspiración potencial anual), en la zona costera desde la VII Región $\left(36^{\circ} \mathrm{S}\right)$ y hasta la VIII Región $\left(38^{\circ} 30^{\prime}\right)$. Para su valoración se usó la información y los rangos de tolerancia para especies de rápido crecimiento (se considera como adecuado un índice de humedad anual de 1 y favorable sobre 1.5), definidos por Schlatter et al. (1994, 1995), y complementada por el Atlas Agroclimático de Santibáñez y Uribe (1993)

\section{RESULTADOS}

REQUERIMIENTOS AMBIENTALES DEL AROMO AUSTRALIANO. El cuadro 1 entrega los promedios de las condiciones climáticas, obtenidas para zonas, distritos y áreas climáticas desde los datos recopilados por Walter y Lieth (1960). El cuadro 2 complementa la anterior información entregando datos climáticos en los límites geográficos de la distribución natural de la especie en el continente (zona A), con el fin de identificar las condiciones umbrales para la especie (ver figura 1).

\section{PRECIPITACION Y DURACION DEL PERIODO SECO} Según Esterhuyse (1985) el aromo australiano es favorecido por un clima temperado, con abundantes precipitaciones, en general sobre $800 \mathrm{~mm}$ al año y bien distribuidas. Sin embargo, una mayor cantidad es más favorable en caso de existir un período seco. Siebert y Cerda (1994) recomiendan seleccionar áreas geográficas con más de 1000 mm para establecer aromo en Chile, sin nieve y con una temperatura media anual de $11-13^{\circ} \mathrm{C}$. CONAF (1995) por otra parte recomienda evitar áreas con 


\section{CUADRO 1}

Promedio de variables climáticas en la distribución natural de Acacia melanoxylon.

Climatic variables average in the natural distribution of Acacia melanoxylon.

\begin{tabular}{|c|c|c|c|c|c|c|c|c|c|c|}
\hline Zona & $\mathrm{TC}$ & $\mathrm{D}$ & $\mathrm{PP}$ & DPS & $\mathrm{T}$ & PLH & $\mathrm{TM}$ & $\mathrm{MT}$ & TMA & MTA \\
\hline \multirow[t]{6}{*}{ A } & I1 & \multirow{6}{*}{$\begin{array}{l}* \\
*\end{array}$} & 1331 & 0 & 18.6 & 339 & 7.8 & 0 & -0.9 & 2.1 \\
\hline & $\mathrm{I} 2$ & & 869 & 0 & 16.0 & 226 & 3.3 & 0 & -5.6 & 6.4 \\
\hline & IV3a & & 543 & $1-4$ & 14.0 & 196 & 3.2 & 0 & -4.9 & 8.2 \\
\hline & IV $3 b$ & & 732 & $0-1$ & 12.5 & 240 & 5.0 & 0 & -3.5 & 5.5 \\
\hline & IV $3 c$ & & 772 & 0 & 14.0 & 256 & 5.2 & 0 & -2.6 & 5.0 \\
\hline & V3 & & 635 & 0 & 14.0 & 167 & 1.7 & 0 & -8.2 & 9.0 \\
\hline B & I1 & & 1718 & 1 & 22.3 & 365 & 11.5 & 0 & 1.7 & 0.0 \\
\hline C y D & I1 & & 2663 & 1 & 23.7 & 365 & 14.2 & 0 & 3.9 & 0.0 \\
\hline $\mathrm{E}$ & IV $1 \mathrm{~b}$ & & 367 & 4 & 14.3 & 170 & 2.5 & 0 & -7.2 & 8.0 \\
\hline $\mathrm{F}$ & IV $2 b$ & & 693 & 4 & 15.0 & 264 & 5.8 & 0 & -2.7 & 4.5 \\
\hline \multirow[t]{4}{*}{ G } & IV $3 b$ & & 698 & $0-1$ & 12.6 & 233 & 4.2 & 0 & -4.2 & 6.5 \\
\hline & IV $3 c$ & $*$ & 868 & 0 & 12.4 & 181 & 7.4 & 0 & -4.0 & 6.5 \\
\hline & V3 & & 586 & 0 & 11.3 & 162 & 2.8 & 0 & -7.6 & 7.5 \\
\hline & $\mathrm{V} 4$ & $*$ & 1866 & 0 & 10.0 & 196 & 3.8 & 0 & -6.1 & 11.8 \\
\hline
\end{tabular}

(Fuente: Walter y Lieth 1960).

TC : Subtipo climático. Los números y letras corresponden a subdivisiones dentro del tipo climático correspondiente.

D : El signo (*) indica donde aromo australiano alcanza desarrollo óptimo según Kannegiesser (1989).

PP : Precipitación media anual $(\mathrm{mm})$.

DPS : Duración del período seco (meses).

PLH : Período libre de heladas (días/año).

$\mathrm{T} \quad$ : Temperatura media anual $\left({ }^{\circ} \mathrm{C}\right)$.

TM : Temperatura media mínima del mes más frío $\left({ }^{\circ} \mathrm{C}\right)$.

MT : Meses con temperatura media mínima bajo $0^{\circ} \mathrm{C}$

TMA : Temperatura mínima absoluta $\left({ }^{\circ} \mathrm{C}\right)$.

MTA : Meses con temperatura mínima absoluta bajo $0^{\circ} \mathrm{C}$.

CUADRO 2

Caracterización climática en los límites de la distribución continental de la especie.

Climatic characterization in the limits of the continental distribution of the species.

\begin{tabular}{|c|c|c|c|c|c|c|c|c|c|c|}
\hline Zona & $\mathrm{TC}$ & $\mathrm{PA}$ & $\mathrm{PP}$ & DPS & $\mathrm{T}$ & PLH & TM & MT & TMA & MTA \\
\hline \multirow[t]{2}{*}{ Norte } & I1 & $\mathrm{NO}$ & 1188 & 0 & 20.7 & 343 & 9.3 & 0.0 & 0.9 & 1.4 \\
\hline & I1 & SI & 1595 & 0 & 19.7 & 352 & 8.1 & 0.0 & 2.1 & 1.5 \\
\hline Centro- & $\mathrm{I} 2$ & $\mathrm{NO}$ & 797 & 0 & 15.2 & 200 & 1.6 & 0.2 & -8.4 & 8.2 \\
\hline Norte & $\mathrm{I} 2$ & SI & 766 & 0 & 16.5 & 224 & 2.7 & 0.0 & -7.7 & 7.0 \\
\hline \multirow[t]{2}{*}{ Centro } & V3 & NO & 660 & 0 & 15.2 & 176 & 0.8 & 0.3 & -9.8 & 8.3 \\
\hline & $\mathrm{I} 2$ & SI & 968 & 0 & 15.7 & 246 & 4.1 & 0.0 & -3.9 & 5.7 \\
\hline \multirow[t]{5}{*}{ Oeste } & IV $1 b$ & NO & 414 & 6 & 15.4 & 215 & 3.7 & 0.0 & -4.5 & 6.8 \\
\hline & IV $3 a$ & $\mathrm{NO}$ & 533 & $4-5$ & 15.7 & 214 & 3.3 & 0.0 & -5.1 & 6.8 \\
\hline & IV $3 a$ & SI & 543 & $2-4$ & 14.0 & 196 & 3.2 & 0.0 & -4.9 & 8.2 \\
\hline & IV $3 b$ & SI & 732 & $1-2$ & 12.5 & 240 & 5.0 & 0.0 & -3.5 & 5.5 \\
\hline & IV $3 c$ & SI & 772 & $0-1$ & 14.0 & 256 & 5.2 & 0.0 & -2.6 & 5.0 \\
\hline
\end{tabular}

(Fuente: Walter y Lieth 1960).

(Abreviaturas de variables climáticas en cuadro 1).

PA: Presencia de la especie en el subtipo climático correspondiente. 
un período seco superior a 6 meses. Harrison (1975) observa que en Australia la especie logra madera de calidad sólo en sitios de condiciones favorables.

En la zona tropical de Australia, la especie es susceptible a un desequilibrio hídrico, ya que su requerimiento anual supera los $1100 \mathrm{~mm}$, por las altas temperaturas y la falta de una período de receso invernal en esa zona. Sin embargo en climas temperados $\left(>30^{\circ} \mathrm{S}\right)$, las exigencias en lluvias anuales disminuyen, calificándose como sitios óptimos aquellos con más de $600 \mathrm{~mm}$, pero sin un período seco definido.

En climas más fríos (Tasmania), las exigencias por agua son menores. En el continente australiano, hacia el Oeste de la distribución de la especie, el umbral necesario desciende a alrededor de 400$500 \mathrm{~mm}$. Sin embargo, si el período seco supera los 4 meses la especie no puede sobrevivir.

REGIMEN DE TEMPERATURA. Existe una clara tendencia a la disminución de la temperatura media con el incremento de la latitud. Los sitios de crecimiento óptimo no presentan una temperatura media anual inferior a $10^{\circ} \mathrm{C}$, umbral que se infirió desde diagramas climáticos. Las temperaturas medias mínimas en general no descienden en ningún mes del año de $0^{\circ} \mathrm{C}$ en donde la especie se desarrolla. Por su parte, la temperatura mínima absoluta no desciende de $-10^{\circ} \mathrm{C}$. Según Siebert y Cerda (1994), temperaturas mínimas absolutas de - $8^{\circ} \mathrm{C}$ son mortales para las plantas de corta edad.

En Australia las temperaturas medias mínimas y mínimas absolutas descienden en forma notoria hacia el interior del continente, como se observa en el cuadro 2, en las áreas geográficas donde la especie deja de crecer.

El período libre de heladas disminuye igualmente con el aumento de la latitud, llegando en algunos sectores elevados de Tasmania a menos de 50 días de duración. Sin embargo, el aromo no sobrevive un período menor a 130 días libre de heladas, en general, dependiendo esto de su procedencia. Por otra parte, en algunos sectores costeros de Australia y Tasmania las heladas pueden estar ausentes en todo el año, lo que favorece a la especie.

El cuadro 3 presenta los rangos de tolerancia a variables climáticas del aromo australiano como resultado del anterior análisis, considerándose para tal efecto regiones sobre $\operatorname{los} 35^{\circ} \mathrm{S}$.

La información bibliográfica y la entregada por los cuadros 1 y 2 permiten identificar las variables climáticas más importantes, entregadas en el cuadro 3 .

REQUERIMIENTOS DE SUELO Y DE UBICACION TOPOGRAFICA.Gleason (1986) menciona que el aromo australiano logra la mejor calidad en suelos profundos y húmedos, en lo posible sobre los 70 $\mathrm{cm}$, y con buen drenaje. Sin embargo, la profundidad depende de las condiciones climáticas y la pedregosidad en el suelo. Menores precipitaciones, un período seco más prolongado y una mayor pedregosidad requieren de un suelo de mayor profundidad (Grey y Taylor 1983).

La especie se desarrolla de mejor calidad como árbol en sitios protegidos, ya sea por su ubicación topográfica o por la presencia de otras especies arbóreas que le dan protección lateral, lo cual es sumamente importante si se desea lograr madera de calidad (Grey y Taylor 1983, Gleason 1986).

El cuadro 4 entrega los niveles cualitativos distinguidos para las variables del suelo, en complemento a las variables climáticas.

Las variables del suelo indicadas son las que pudieron identificarse como las más importantes desde la literatura accesible, dando énfasis a las condiciones óptimas para el crecimiento de la especie.

DISTRIBUCION POTENCIAL DE AROMO AUSTRALIANO EN CHILE. La figura 2 muestra la distribución potencial en Chile para el establecimiento de aromo australiano y, a manera de referencia, se indican algunos códigos de distritos de la zona de crecimiento costa de acuerdo a los utilizados por el Sistema de Ordenamiento de la Tierra (Schlatter et al. 1994 y 1995). El límite norte de la distribución potencial, para lograr bosques de buen potencial de crecimiento, se encuentra principalmente en la VIII Región. Pero además es posible su establecimiento puntual en sectores ubicados en las VII y VI Regiones, tanto en la costa como en la precordillera, en ubicación de pie de monte (más húmedos) y/o en sitios de suelos profundos de alta capacidad de agua aprovechable.

Hacia el sur la distribución abarca hasta Chiloé, siguiendo desde la latitud $42^{\circ} \mathrm{S}$ una franja costera y de poca altitud, hasta la XI Región.

Existen amplias zonas con calidades de sitio de Categoría 1 en sectores costeros e interiores desde el $\mathrm{N}$ de la IX Región hasta el $\mathrm{S}$ de la provincia de Llanquihue, en la X Región. Algunas zonas de Categoría 2 es posible encontrar en este mismo tramo (limitaciones puntuales en el suelo). 


\section{CUADRO 3}

Rangos de tolerancia de variables climáticas estimados para Acacia melanoxylon. Range of climatic variable tolerance estimated for Acacia melanoxylon.

\begin{tabular}{|c|c|c|c|}
\hline Factor climático & Unidad & Criterio & Rango estimado \\
\hline \multirow[t]{4}{*}{ Precipitación } & \multirow[t]{4}{*}{$\mathrm{mm}$} & Favorable & Más de 800 mm \\
\hline & & Adecuado & 626 a $800 \mathrm{~mm}$ \\
\hline & & Moderado & 451 a $625 \mathrm{~mm}$ \\
\hline & & Desfavorable & Menos de $450 \mathrm{~mm}$ \\
\hline \multirow[t]{4}{*}{ Duración período seco } & \multirow[t]{4}{*}{ meses } & Favorable & Sin período seco \\
\hline & & Adecuado & 0.1 a 1.9 meses \\
\hline & & Moderado & 2.0 a 3.9 meses \\
\hline & & Desfavorable & Más de 4 meses \\
\hline \multirow[t]{4}{*}{ Período libre de heladas } & \multirow[t]{4}{*}{ días } & Favorable & Más de 300 días \\
\hline & & Adecuado & 231 a 300 días \\
\hline & & Moderado & 161 a 230 días \\
\hline & & Desfavorable & Menos de 160 días \\
\hline \multirow[t]{4}{*}{ Temperatura media anual } & \multirow[t]{4}{*}{${ }^{\circ} \mathrm{C}$} & Favorable & Más de $12.4^{\circ} \mathrm{C}$ \\
\hline & & Adecuado & 9.8 a $12.4^{\circ} \mathrm{C}$ \\
\hline & & Moderado & 7.1 a $9.7^{\circ} \mathrm{C}$ \\
\hline & & Desfavorable & Menos de $7^{\circ} \mathrm{C}$ \\
\hline \multirow{4}{*}{$\begin{array}{l}\text { Temperatura media } \\
\text { mínima del mes más frío }\end{array}$} & \multirow[t]{4}{*}{${ }^{\circ} \mathrm{C}$} & Favorable & Más de $4.4^{\circ} \mathrm{C}$ \\
\hline & & Adecuado & 2.3 a $4.4^{\circ} \mathrm{C}$ \\
\hline & & Moderado & 0.1 a $2.2^{\circ} \mathrm{C}$ \\
\hline & & Desfavorable & Menos de $0^{\circ} \mathrm{C}$ \\
\hline
\end{tabular}

\section{CUADRO 4}

Criterio de evaluación de variables edáficas estimadas para Acacia melanoxylon.

Criterion of estimated soil variable evaluation for Acacia melanoxylon.

\begin{tabular}{|lll|}
\hline \multirow{2}{*}{ Factor edáfico } & \multicolumn{2}{c|}{ Criterio } \\
\cline { 2 - 3 } Profundidad & Favorable & Desfavorable \\
& $\begin{array}{l}\text { Suelo profundo a muy profundo, } \\
\text { rico en materia orgánica }\end{array}$ & $\begin{array}{l}\text { Suelo de escasa profundidad, } \\
\text { Suelo no desarrollado (rocoso), } \\
\text { Arraigabilidad }\end{array}$ \\
Capacidad de campo & Media a alta & Suelo turboso \\
Textura & Media a alta, agua aprovechable & Baja \\
Estructura & Franca, franca limosa & Extrema (predominancia de arenas \\
& Estructuras sueltas, de tipo grumoso & Estructuras compactas \\
Drenaje interno & o granular & Suelos de drenaje restringido o \\
& Suelo bien drenado & impedido. Suelos con \\
& & anegamiento prolongado
\end{tabular}




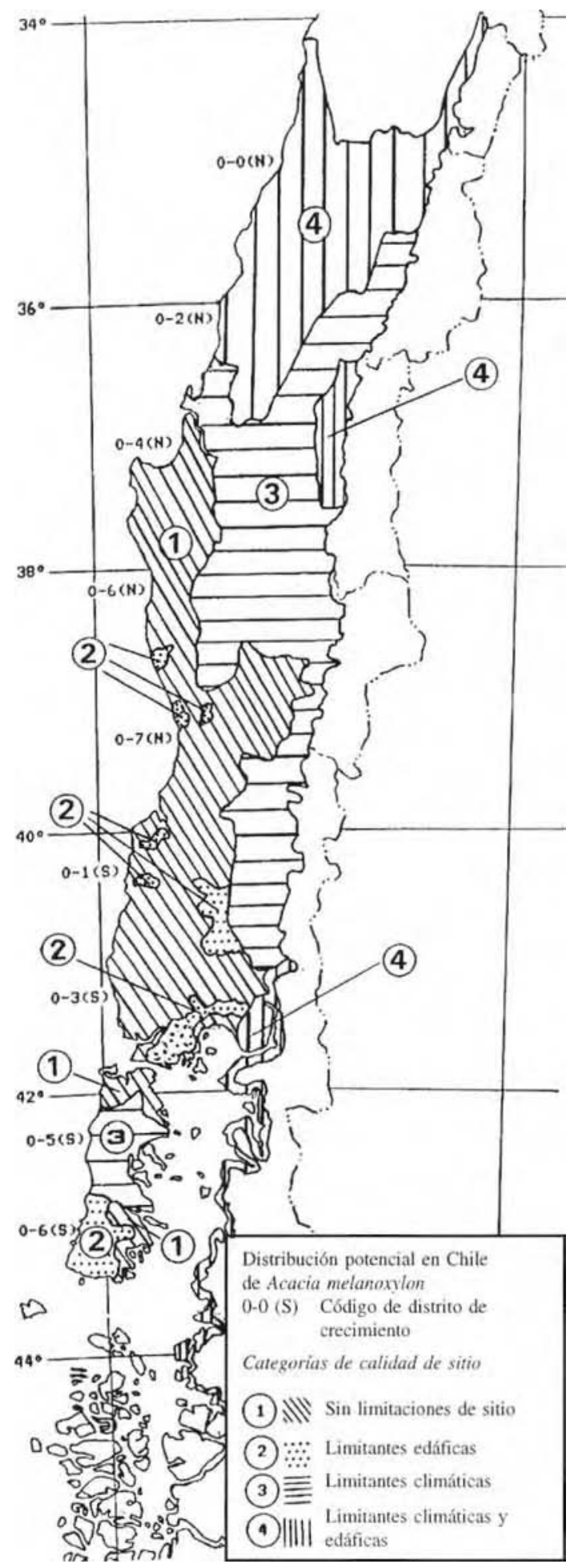

Figura 2. Distribución potencial y estimación de calidad de sitio para Acacia melanoxylon.

Potential distribution and quality of site estímate for Acacia melanoxylon.
La mayor parte del tramo costero desde $\operatorname{los} 37^{\circ} \mathrm{S}$ hasta el sur de la VIII Región corresponde originalmente a zonas de Categoría 3 (período seco fuera del rango de tolerancia de la especie). Sin embargo, los distritos de crecimiento de la costa de la VIII Región, luego de aplicar el criterio de corrección (efecto oceánico, ver método), son transformados a Categoría 1. Es decir, al reemplazar el período seco por el de índice de humedad anual como criterio de discriminación, las variables de sitio analizadas están todas en los rangos de tolerancia de la especie.

La franja precordillerana de las Regiones IX y $X$ son en su totalidad de Categoría 3 por el efecto de la temperatura invernal (se agrega al período seco en el norte). Por su parte, la zona costera, desde la latitud $42^{\circ} \mathrm{S}$ y hasta $\operatorname{los} 44^{\circ} 30^{\prime} \mathrm{S}$ (por limitaciones de temperatura y suelo), como también el extremo norte de la distribución potencial (falta de precipitaciones y período seco prolongado), corresponden a sectores de Categoría 4.

VARIACION DE PUNTAJES DE CALIDAD DE SITIO POR ZONA DE CRECIMIENTO. Las figuras 3 a la 6 muestran la variación en puntos (calidad de sitio) en la distribución potencial de la especie. Se entregan los puntos aportados por las variables climáticas, del suelo y la suma total, para cada distrito de crecimiento. A manera de referencia, en cada figura se indican los distritos de crecimiento, ordenados de $\mathrm{N}$ a $\mathrm{S}$, según el código utilizado por Schlatter et al. (1994, 1995).

ZONA DE CRECIMIENTO COSTA. En esta zona (figura 3) los puntos de las variables del suelo se mantienen relativamente homogéneos. Los mejores puntos se encuentran en la provincia de Valdivia (distrito 0-0(S)). Hacia el extremo sur (Agroclimas 7.31 y 7.21, según el código del Atlas Agroclimático del INIA, 1989) se encuentran los puntos más bajos, correspondientes a áreas de Categoría 4 (con limitaciones climáticas y del suelo).

En cuanto a las variables climáticas de esta zona, la mayoría de los distritos abarcados, hasta el sur de la IX Región, son originalmente de Categoría 3, ya que la duración del período seco es mayor a lo tolerado por la especie. Sin embargo, estos distritos son considerados de Categoría 1, luego de aplicar el criterio de corrección (ver método). En la misma figura se destaca que la corrección produce un aumento notorio en la suma de puntos de los distritos de crecimiento ubicados en la costa 


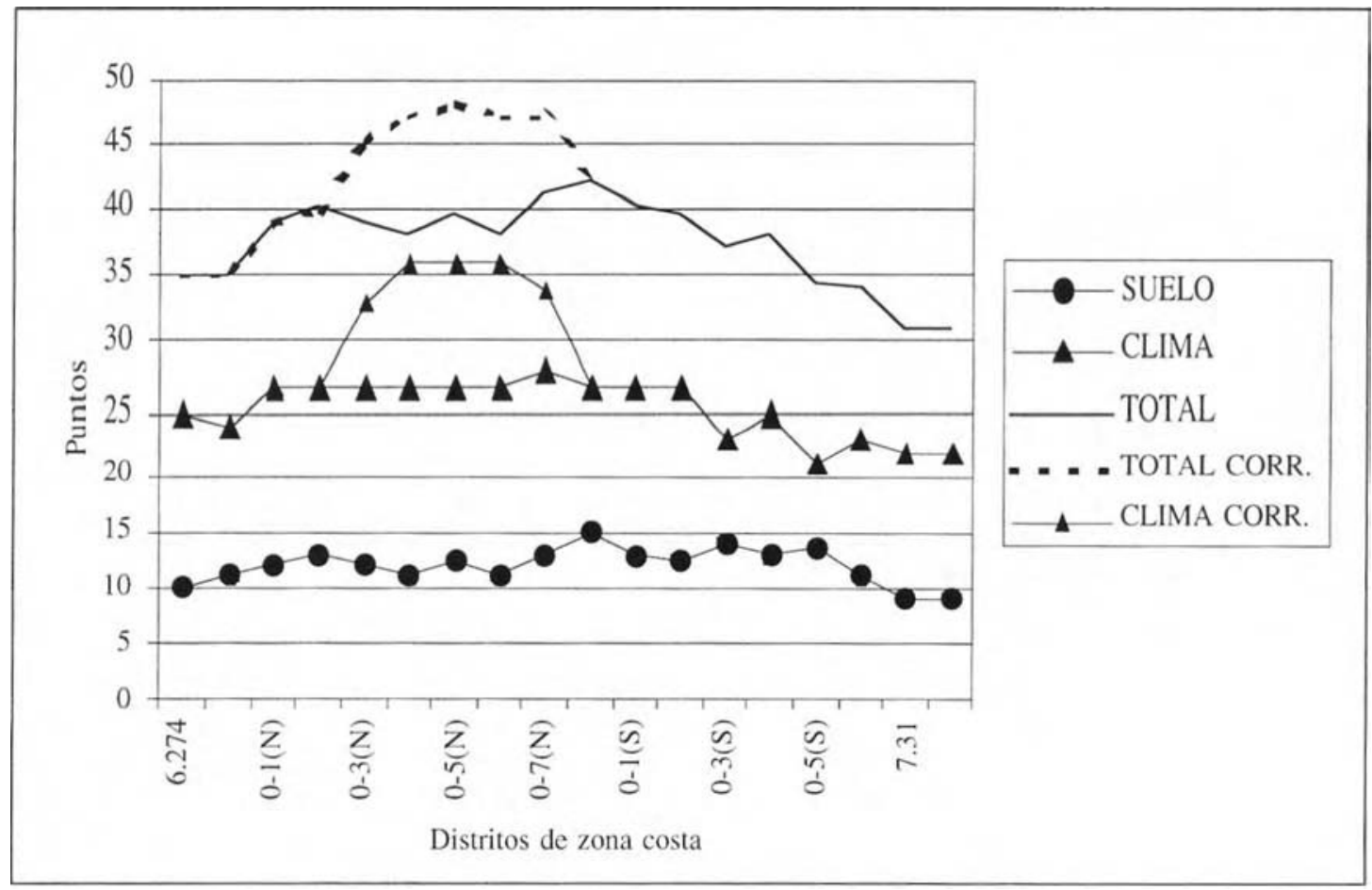

Figura 3. Variación de puntajes de calidad de sitio en zona de crecimiento costa. Variation of site quality scores in the coastal growth zone.

de la VIII Región. A partir de la latitud $42^{\circ} \mathrm{S}$ los puntos por el factor climático descienden notoriamente, siendo los puntos más bajos de esta zona de crecimiento.

La curva del total de puntos (calidad de sitio) es variable en su distribución. Los mejores valores de sitio (igual o superior a 40 puntos) se encuentran en la costa de la VIII Región (sin considerar el período seco). Sin embargo, la costa de la $X$ Región, y hasta cerca del sector de Maullín, alcanza cerca de 40 puntos, descendiendo paulatinamente hasta cerca de 30 en el extremo $S$ de la distribución potencial.

ZONA DE CRECIMIENTO SECANO. En la figura 4 se observa que la calidad del suelo muestra una tendencia a mejorar hacia el $\mathrm{S}$, en cambio las condiciones climáticas se mantienen en un rango de 19-26 puntos. La caída en el distrito 1-7 se debe a un período seco muy prolongado.

El resultado es que en general los mejores sitios para aromo se encuentran en el sur (X Región). En el norte el período seco es la mayor limitación.

ZONA DE CRECIMIENTO VALLE. En la figura 5 se observa que tanto el clima como el suelo presen- tan la mayor cantidad de puntos en el sur. La calidad de sitio tiende a aumentar de $\mathrm{N}$ a S. Hasta el distrito 2-6(N), los puntos promedio son unos 32 . A partir de este sector, aumenta hasta encontrar las mayores sumas de puntos de la zona desde el límite $\mathrm{N}$ de la X Región hasta el lago Llanquihue, bordeando los 40 puntos.

ZONA DE CRECIMIENTO PRECORDILLERA. En la figura 6 se observa que los puntos de las variables del suelo tienden a ser mayor en las áreas centrales de la distribución potencial, por la presencia de suelos trumao, generalmente profundos. Al sur del lago Llanquihue la calidad del suelo decae (relieve accidentado, suelos muy delgados y de textura más gruesa). Los puntos logrados para las variables climáticas son muy fluctuantes en esta zona de crecimiento. Los extremos del N (VI Región) y del $S$ (provincia de Palena) suman pocos puntos. El valor aumenta desde la VII Región hasta la latitud de la ciudad de Temuco (distrito 3-6(N)), exceptuando la zona centro-norte de la VIII Región, donde los puntos vuelven a descender.

Los mayores valores por variables climáticas se encuentran entre la latitud de la ciudad de Temuco y el lago Llanquihue, exceptuando el distrito 3-0(S) (alrededor del lago Raneo, por su 


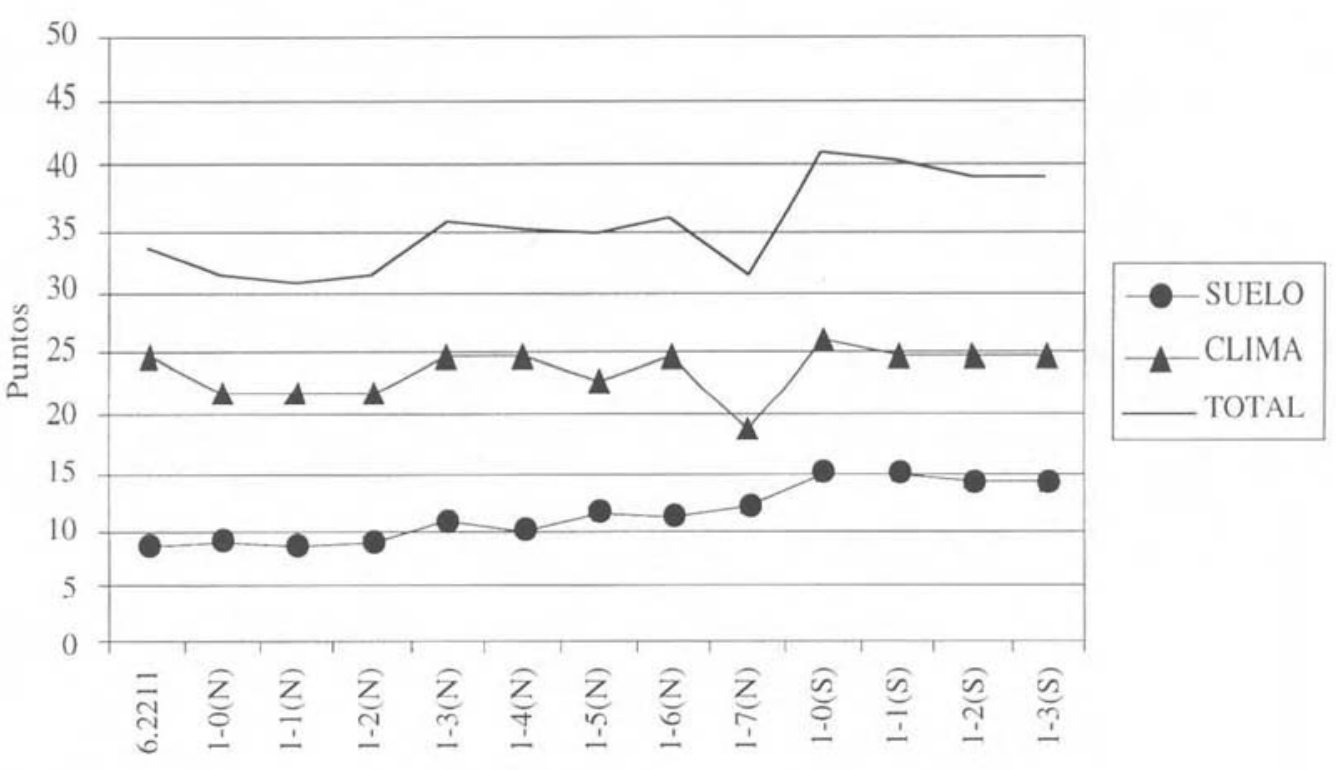

Distritos de zona secano

Figura 4. Variación de puntajes de calidad de sitio en zona de crecimiento secano. Variation of the site quality of scores in the dry land growth zone.



Distritos de Zona depresión intermedia

Figura 5. Variación de puntajes de calidad de sitio en zona de crecimiento depresión intermedia. Variation of the site quality scores in Central-Valley growth zone. 


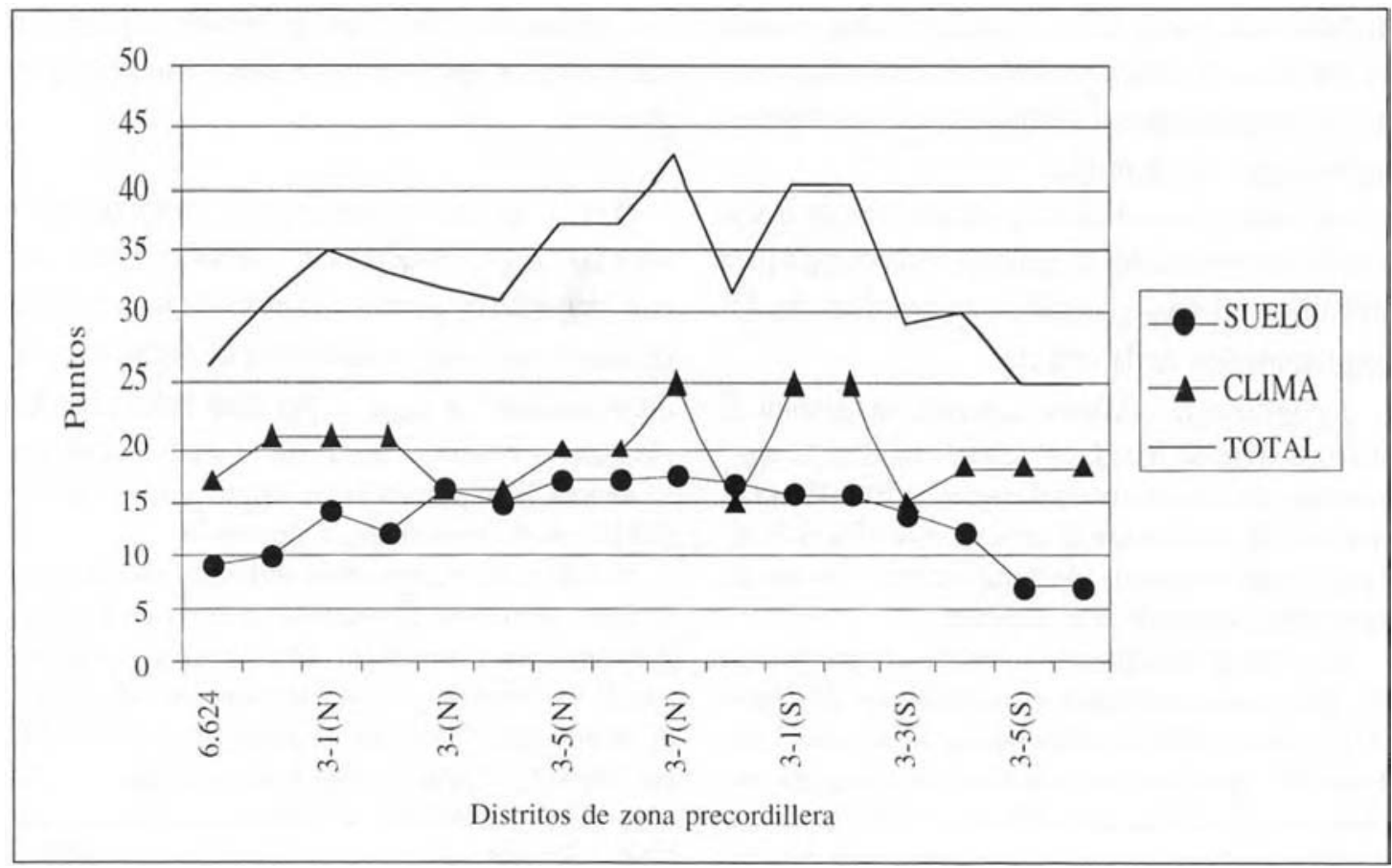

Figura 6. Variación de puntajes de calidad de sitio en zona de crecimiento precordillera. Variation of the site quality scores in the pre-mountain growth zone.

ubicación más cordillerana), donde desciende drásticamente. En los distritos precordilleranos tienden a dominar las categorías 3 y 4 (limitaciones de clima y suelo).

La curva del total de puntos (calidad de sitio) presenta una tendencia errática, similar a la curva de puntos por variables climáticas, encontrándose los valores más bajos en los extremos de la zona (menos de 30 puntos), los que a su vez son los más bajos de toda la distribución potencial de la especie. Al norte el período seco y al sur la temperatura, son los factores más limitados.

Las mejores calidades de sitio (sobre 40 puntos) se dan entre las latitudes $39^{\circ} \mathrm{S}-41^{\circ} \mathrm{S}$ aproximadamente, exceptuando los alrededores del lago Ranco.

\section{DISCUSION}

El Acacia melanoxylon R. Br. presenta su mejor desarrollo en climas templados y húmedos, de precipitaciones bien distribuidas (Harrison 1975). En climas tropicales y subtropicales de Australia el aromo es muy susceptible a un período seco, por las elevadas temperaturas en todo el año en esa región. En ella las lluvias tienden a concen- trarse en la época estival, es decir, la ausencia de la especie en esa región climática, con la excepción de algunos enclaves, se explica por la presencia de un período seco y altas temperaturas (Esterhuyse 1985). Los siguientes bosques de aromo australiano no se encuentran sino a $200 \mathrm{~km}$ al S. La falta de aromo en el tramo mencionado puede deberse a que la humedad resulta insuficiente para compensar las altas temperaturas, lo que origina un déficit hídrico, pues la especie a latitudes $<30^{\circ} \mathrm{S}$ requiere precipitaciones mayores a 1100-1500 mm, y períodos secos menores a dos meses (Ramírez 1997).

La exigencia en precipitaciones es menor con el aumento de la latitud. Al sur de $\operatorname{los} 30^{\circ} \mathrm{S}$ las precipitaciones no disminuyen en general de 500 a $600 \mathrm{~mm}$. Sin embargo, la especie no prospera donde existe un período seco prolongado, sobre 4 meses (Esterhuyse 1985). La información entregada por CONAF (1995) coincide con lo anterior, recomendando evitar el establecimiento de la especie en climas semiáridos, es decir donde existen más de 6-7 meses con déficit pluviométrico. La diferencia de 4 a 6 meses entre Chile y Australia se explica porque por una parte la pluviometría en Chile, donde se inicia el déficit de 6 meses $\left(37^{\circ} \mathrm{S}\right)$, es de 1000-1200 mm y, por otra, el clima es muy 
influenciado por el efecto oceánico, compensando el efecto de un déficit de lluvia. Además, los terrenos en general presentan suelos profundos de buena retención de humedad.

La caída pluviométrica y el período seco son por lo tanto variables de primera importancia para identificar el área geográfica compatible con los requerimientos de la especie.

La influencia oceánica aumenta en general el período libre de heladas y además modera la temperatura media mínima del mes más frio. Hacia el interior del continente la falta de este efecto oceánico causa extremos de temperatura y falta de humedad, limitando a la especie.

La especie desaparece si existe algún período del año con temperatura media mínima inferior a $0^{\circ} \mathrm{C}$, aun cuando la temperatura media anual sea favorable, siendo ésta la tendencia más clara encontrada en la literatura (Ramírez 1997). En las áreas geográficas con estas limitaciones, la temperatura mínima absoluta desciende a $-8.4^{\circ} \mathrm{C}$ e incluso menos, situación considerada como umbral por Siebert y Cerda (1994). Todo lo anterior confirma los análisis de Esterhuyse (1985), respecto a la gran importancia de la variable temperatura en el desarrollo de la especie.

La temperatura media en su rango óptimo para la especie fluctúa entre $10-15^{\circ} \mathrm{C}$, de acuerdo al análisis efectuado por Ramírez (1997). Esta variable disminuye con el aumento de la latitud, y con ella se acorta el período vegetativo y se favorece un período de latencia vegetativa más prolongado, lo cual, de acuerdo a Harrison (1975), favorece la calidad de la madera. Esto coincide con las zonas definidas como mejores por Kannegiesser (1989), es decir, al sureste de Australia y noroeste de Tasmania.

En cuanto al suelo, las variables más destacadas por la literatura, para definir la fertilidad de un suelo en climas con déficit hídrico, son las relacionadas con el régimen de agua (Ramírez 1997). Entre éstas, las características como la profundidad de desarrollo del suelo, la textura y la estructura son fundamentales pues definen propiedades como la posibilidad de arraigamiento, la capacidad de agua aprovechable y el drenaje interno. Esto ha sido comprobado en la literatura, especialmente en esludios relacionados con las plantaciones de Pinus radiata en Chile (Schlatter et al. 1982, Schlatter 1993, Schlatter y Gerding 1995, Bonelli y Schlatter 1995). De los análisis de la literatura relacionada con el aromo, estas variables también son destacadas como las de mayor importancia para estudios que cubren amplios espacios geográficos.

\section{EFECTO DE LAS VARIABLES DE SITIO EN EL DESA-} RROLLO DE LA ESPECIE. Las variables climáticas más importantes para la estimación de la calidad de sitio se agrupan en aquellas relacionadas con la disponibilidad de agua y, por otro lado, las relacionadas a la temperatura. En el análisis anterior se denota la importancia de ambos grupos de variables en el crecimiento y desarrollo.

El valor de precipitación utilizado para definir el sitio óptimo en el presente análisis es el recomendado por Esterhuyse (1985) para especies de rápido crecimiento, en latitudes mayores a $35^{\circ} \mathrm{S}$, y es de $800 \mathrm{~mm}$. Este valor es menor al mencionado por Siebert y Cerda (1994) para establecer aromo australiano (alrededor de $1000 \mathrm{~mm}$ anuales) en Chile. Sin embargo, esta desigualdad es posible explicarla por las diferencias climáticas entre ambos países. En Chile el período seco es más prolongado en las áreas geográficas con precipitaciones menores a $800 \mathrm{~mm}$ que en Australia, generalmente mayor a 7 meses $\left(<36^{\circ} \mathrm{S}\right)$. En esas condiciones el aromo puede desarrollarse sólo en condiciones puntuales con suelos muy profundos y de alta capacidad de agua aprovechable, o bien en posición de pie de monte y/o en la zona costera con influencia oceánica directa.

El factor temperatura media presenta un rango estrecho para la estimación de la calidad de sitio; sin embargo, es complementado por la evaluación del período libre de heladas y temperatura media mínima, factores que demostraron las más claras tendencias con la distribución natural, y que permiten una mejor aproximación de las condiciones umbrales de un sitio.

LA CALIDAD DE SITIO PARA AROMO EN CHILE. La distribución potencial de la especie, delimitada y analizada en este trabajo principalmente con fines productivos, precisa la estimación de CONAF (1995), que no diferenció entre sitios para la sobrevivencia y sitios aptos para producción maderera. Por cierto que es deseable aprovechar las características ecológicas del aromo australiano, estableciéndolo en terrenos donde sea necesaria la recuperación de suelos y el control de dunas. La especie puede sobrevivir en sitios con condiciones poco favorables para su desarrollo, ya que presenta una particular capacidad de resistir un 
medio adverso (Forest Research Institute, 1982; Kannegiesser 1989). Sin embargo, en tales condiciones no puede en general esperarse madera de calidad.

En el norte de su distribución potencial en Chile, la principal restricción para el desarrollo del aromo es la longitud e intensidad del período seco. El nivel de precipitaciones, en cambio, según los rangos de tolerancia definidos para Australia, no constituye una restricción. Lo anterior es aún más grave si el suelo no posee una alta capacidad de retención de agua, que pueda cubrir la demanda de la especie durante su período de crecimiento. Esto también es válido para los suelos de arena que se encuentran al interior de la VIII Región, cuya capacidad de agua aprovechable impide el desarrollo del aromo, a pesar de existir en esa zona precipitaciones de 1200-1500 $\mathrm{mm}$ anuales. En estos suelos presenta en cambio un buen crecimiento cuando en el subsuelo aparece agua en movimiento permanente.

Las temperaturas mínimas y el período de heladas, y la intensidad de éstas, son las principales restricciones climáticas que se encuentran en el sur de la distribución potencial de la especie. Los sectores costeros tienen temperaturas moderadas debido al efecto del océano; sin embargo, este efecto va disminuyendo hacia el interior del continente y hacia mayores elevaciones, encontrándose las más grandes limitaciones de temperatura en los distritos de la zona de crecimiento precordillera (Schlatter et. al. 1994 y 1995).

En general, los mejores sitios para el establecimiento de aromo se encuentran en la zona de crecimiento costa, desde la zona de Concepción $\left(37^{\circ} \mathrm{S}\right)$, hasta el norte y este de Chiloé $\left(42^{\circ} 30^{\prime} \mathrm{S}\right.$, aproximadamente). Desde el punto de vista de la distribución de las precipitaciones, el mejor crecimiento es al sur de la X Región. Sin embargo, bajo los $400 \mathrm{~m}$, cerca del litoral en las IX y VIII Regiones, se presenta un alto potencial para la especie, gracias al efecto oceánico, especialmente en verano. Las áreas que Siebert y Cerda (1994) estiman como más favorables para la especie concuerdan con estos resultados.

El estudio de INFOR (1986) también estima que la zona costera de Arauco es una de aquellas con la más alta potencialidad para la producción de aromo, pero lamentablemente no especifica los factores ambientales que lo posibilitan. El trabajo anterior, al igual que Cerda (1995), describe como la zona más favorable aquella que se extiende desde la costa al N de la X Región $\left(39^{\circ}-41^{\circ} \mathrm{S}\right)$, hasta la precordillera en dirección este. Tal aproximación coincide con el presente trabajo. La homogeneidad de la calidad de sitio en esta última zona se debe a la compensación paulatina de las variables climáticas más desfavorables hacia la precordillera por el aumento en esa dirección de la calidad del suelo. Esta zona está cubierta principalmente por los llamados suelos trumao, de origen volcánico, profundos y muy fértiles para la producción forestal (Schlatter et al. 1994 y 1995).

\section{CONCLUSIONES}

Acacia melanoxylon $\mathrm{R}$. Br. en Australia puede alcanzar su óptimo desarrollo en climas templado-húmedos cuyas características son precipitaciones de $800 \mathrm{~mm}$ o superiores, período seco inferior a 4 meses, temperatura media mensual entre 10-15 C, más de 150 días libres de heladas, temperaturas mínimas absolutas que no bajen de los $-8^{\circ} \mathrm{C}$ y temperaturas mínimas medias mensuales sobre $0^{\circ} \mathrm{C}$.

E1 suelo que el aromo requiere debiera ser de más de $70 \mathrm{~cm}$ de profundidad posible de arraigar, de texturas medias a finas y bien estructurado, de alta capacidad de agua aprovechable y buen drenaje. Esto coincide con el óptimo requerido para la mayoría de las especies forestales.

La calidad de la madera es favorecida si la especie entra en un tipo de receso invernal, de preferencia medio a prolongado. La precisión de este factor sin embargo es insuficiente en la literatura.

La especie también puede desarrollarse en climas subtropicales y tropicales $\left(<30^{\circ} \mathrm{S}\right)$, pero con precipitaciones sobre los 1100-1500 mm anuales, y sin un período seco mayor a 2 meses. En esas regiones climáticas sólo aparece en enclaves costeros, de alta humedad atmosférica y de temperaturas más moderadas.

En zonas templadas y templado-frías la especie se desarrolla bien con más de $600 \mathrm{~mm}$ de precipitaciones, pero necesita al menos 400-500 mm para sobrevivir, lo que es imposible con un período seco mayor a 4 meses, en zonas de clima continental.

Entre Australia y Chile existen diferencias climáticas que hacen distintos algunos valores umbrales de exigencia del aromo. En Chile Central el período seco es más prolongado para equivalentes precipitaciones en Australia. Por tal moti- 
vo la pluviometría mínima necesaria en Chile para un buen crecimiento es superior en comparación a Australia. Por otra parte, el efecto oceánico en la zona costera de Chile modera considerablemente el período seco y la temperatura, favoreciendo su sobrevivencia en distritos con períodos secos mayores a 6 meses.

En Chile, el límite norte de la distribución potencial de la especie, para fines de producción maderera, se encuentra en la VIII Región ( $\left.37^{\circ} \mathrm{S}\right)$. Su desarrollo también es posible en algunas localidades costeras y precordilleranas ubicadas al norte del límite indicado, donde la ubicación topográfica y la fertilidad del suelo puedan compensar la insuficiencia de precipitaciones durante un prolongado período seco. Pero en esas zonas deben seleccionarse rigurosamente los sitios aptos, y mientras más largo el período seco, mayor el riesgo.

El límite sur de la distribución potencial se encuentra en la latitud $42^{\circ} 30^{\prime} \mathrm{S}$, aproximadamente. Más al sur su desarrollo es posible en una franja costera de poca altitud, con suelos profundos. Su establecimiento en esta distribución geográfica se ve limitado por las temperaturas mínimas y el prolongado período con heladas.

En Chile también es posible aprovechar la gran capacidad del aromo australiano de colonizar y recuperar suelos degradados. La especie tiene capacidad de resistir en medios más adversos a los indicados como umbrales para su óptimo desarro1lo, y probablemente existan procedencias más resistentes. En estos casos no se debe sin embargo evaluar su crecimiento con criterio de rentabilidad para la producción de madera.

Los sitios más adecuados para el establecimiento de la especie, con fines de producción, se encuentran en general bajo los 400 m s.n.m., en la zona costera de la VIII Región, y en la costa y zonas interiores de las IX y X Regiones, hasta el norte y este de la Isla de Chiloé. Sin embargo, deben evitarse sitios precordilleranos con temperaturas umbrales y además, para obtener árboles de calidad, se deben evitar los sitios que tengan limitaciones severas de suelo, como poca profundidad, baja retención de agua o mal drenaje.

El aromo australiano además es favorecido en su calidad en ubicaciones topográficas poco expuestas al viento y a la nieve, y es agradecida a la protección lateral de otras especies.

\section{BIBLIOGRAFIA}

BOLAND, D.J., M.I.H. BROOKER, G.M. CHIPPENDALE, N. HALL, B.P.M. HYLAND, R.D. JOHNSTON, D.A. KLEINIG, J.D. TURNER. 1992. Forest trees of Australia. CSIRO Publishing, Victoria, Australia, 687 p.

BONELLI, C, J.E. SCHLATTER. 1995. "Caracterización de suelos rojo arcillosos de la zona Centro-Sur de Chile". Bosque 16(2): 21-37.

CERDA, J.P. 1995. Productividad de Acacia melanoxylon R. Br. (aromo australiano) en relación con algunos sitios. Tesis, Fac. Ciencias Forestales, Univ. Austral de Chile, Valdivia, $81 \mathrm{p}$.

CONAF 1991. "Aromo australiano, ¿una especie del futuro? I Parte", Chile Forestal 180:28-29.

CONAF 1995. Aromo australiano. Folleto divulgación, Corporación Nacional Forestal, Santiago, Chile.

DE ZWAAN, J.G. 1982. "The silviculture of Blackwood", South African Forestry Journal 113: 59-61.

ESTERHUYSE, C.J. 1985. "Site requirements of the most important commercial trees planted in South Africa", South African Forestry Journal 133: 61-66.

FARRELL, T.P., D.H. ASHTON. 1978. "Population studies on Acacia melanoxylon $\mathrm{R}$. Br. Variation in seed and vegetative characteristics", Aust. .J. Bot. 26: 365-379.

FOREST RESEARCH INSTITUTE, 1982. "Australian Blackwood (Acacia melanoxylon)", What's new in forest research? 105, $4 \mathrm{p}$.

GLEASON, C.D. 1986. "Tasmanian Blackwood, it's potential as a timber species", New Zealand Journal of Forestry 6-12.

GREY, D.C., G.I. TAYLOR. 1983. "Site requirements for commercial afforestation in the Cape", South African Forestry Journal 127: 35-38.

HARRISON, C.M. 1975b. "Heartwood colour patterns in South Africa Acacia melanoxylon", Forestry in South Africa 17: 49-56.

INIA 1989. Mapa agroclimático de Chile. Instituto de Investigaciones Agropecuarias, Ministerio de Agricultura, Santiago de Chile, $221 \mathrm{p}$.

INSTITUTO FORESTAL 1986. Especies forestales exóticas de interés económico para Chile. INFOR, CORFO, Santiago, Chile, 168 p.

KANNEGIESSER, U. 1989. "Antecedentes generales sobre Acacia melanoxylon (aromo australiano)", Ciencia $e$ investigación forestal 2(7): 90-97.

LAGOS, E. 1994. "Diversificando Chile", Chile Forestal 216: 28-30.

SANTIBAÑEZ, F., J.M. URIBE. 1993. Atlas Agroclimático de Chile. Santiago (Chile), Fondo Nacional de Desarrollo Científico y Tecnológico, 99 p.

SCHLATTER, J.E. 1993. Fertilidad del suelo, concepto y su aplicación a la producción forestal. Charlas y Conferencias $\mathrm{N}^{\circ} 8$, Fac. Ciencias Forestales, Univ. Austral de Chile, 78-86.

SCHLATTER, J.E., V. GERDING, M. BONNEFOY. 1982. Factores del sitio de mayor incidencia en la productividad de Pinus radiata D.Don. En: Actas de la Reunión de Trabajo sobre Evaluación de la Productividad de Sitios Forestales, Fac. Ciencias Forestales, Univ. Austral de Chile: 61-97.

SCHLATTER, J.E., V. GERDING, J. ADRIAZOLA. 1994. Sistema de ordenamiento de la tierra, herramienta para la planificación forestal aplicada a las regiones VII, VIII y IX. Serie Técnica. Fac. Ciencias Forestales, Univ. Austral de Chile, $114 \mathrm{p}$.

SCHLATTER, J.E., V. GERDING, H. HUBER. 1995. Sistema de ordenamiento de la tierra, herramienta para la planificación forestal aplicada a la X Región. Serie Técnica, Fac. Ciencias Forestales, Univ. Austral de Chile, 93 p. 
SIEBERT, H., J.P. CERDA. 1994. Aspectos prácticos de la silvicultura del aromo australiano. Tercer taller silvícola "Diversificación y silvicultura". Santiago (Chile), pp. 5-16. RAMIREZ, J.C. 1997. Determinación del área potencial para el establecimiento en Chile de Acacia melanoxylon $\mathrm{R}$. Br. Tesis, Fac. Ciencias Forestales, Univ. Austral de Chile, Valdivia, $105 \mathrm{p}$
WALTER, H., A. LIETH. 1960. Klimadiagramm Weltatlas. Jena. (VEB) Gustav Fischer Verlag, s.p.

WILKINS, A.P., S.E. PAPASSOTIRIOU. 1989. "Wood anatomical variation of Acacia melanoxylon in relation to latitude", IAWA-Bulletin 10(2): 201-207. 